\title{
ALGUNOS ASPECTOS DE LA LÓGICA JURÍDICA DE LUIGI FERRAJOLI*
}

\author{
Giovanni Battista Ratti ** \\ Universitat de Girona
}

RESUMEN. El artículo presenta y analiza algunos aspectos relevantes del tratamiento de la lógica jurídica ofrecido por L. FERRAJOLI. En particular, el artículo contiene: a) la reconstrucción, desde tres perspectivas diferentes (teoría jurídica, órdenes jurídicos y ciencia jurídica), de los fundamentos del sistema lógico de FerRajoli; $b$ ) el examen de los postulados de completitud y consistencia que desarrollan un papel básico en su teoría axiomática, y finalmente $c$ ) algunas observaciones críticas acerca de la concepción de las normas lógicamente derivadas defendida por FERRAJOLI.

Palabras clave: L. FERRAJOLI, teoría axiomatizada del Derecho, completitud, consistencia, lógica de normas, lógica de proposiciones normativas.

ABSTRACT. The paper aims at presenting and analyzing some relevant features of L. FERRAJOLI's treatment of legal logic. In particular, the paper provides: a) the reconstruction, from three different perspectives (legal theory, legal orders, and legal science), of the foundations of FerRAJoli's logical system; $b$ ) the examination of the postulates of completeness and consistency which are foundational of his axiomatic theory; and finally c) some critical remarks on FERRAJOLI's conception of logically entailed norms.

Keywords: L. FeRRAJOLI, axiomatic theory of Law, completeness, consistency, logic of norms, logic of normative propositions.

\footnotetext{
* Fecha de recepción: 8 de noviembre de 2010. Fecha de aceptación: 10 de diciembre de 2010.

** Investigador «Juan de la Cierva», Universitat de Girona, giovanni.ratti@udg.edu.

Una primera versión, en inglés, de este trabajo fue presentada al seminario «The Axiomatic Construction of a General Theory of Law: discussing Luigi Ferrajoli's Principia Iuris», que tuvo lugar en la Facultad de Derecho de la Universidad de Edimburgo el 31 de mayo 2010, y fue organizado por Paolo Sandro, al cual quiero aquí dar las gracias por haberme brindado la oportunidad de discutir mis ideas en un ambiente tan estimulante. Quisiera además agradecer a Álvaro Núñez por sus valiosas correcciones tanto a la forma como al contenido
} de este ensayo. 


\section{INTRODUCCIÓN}

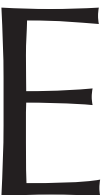

n 1970, el mismo año de la muerte de B. Russell, L. FerRAJOLI publicaba su libro Teoria assiomatizzata del diritto ${ }^{1}$, el primer paso de su completa axiomatización de la teoría del Derecho. Treinta y siete años más tarde, FERRAJOLi ha publicado su magnum opus, que lleva el título - muy russelliano en el espíritu- de Principia Iuris ${ }^{2}$. Esta última obra comparte con la filosofía del «primer» RUSSELL el propósito del «logicismo» ${ }^{3}$ : es decir, la idea de reducir un cierto ámbito de conocimiento a un conjunto restringido de axiomas fundamentales, a desarrollar por medio de deducción estricta. Como es sabido, GöDEL demostró formalmente que el sueño logicista no había sido alcanzado por los Principia Mathematica de Russell y WHITEHEAD, y - lo que es más relevante_- que no podía ser alcanzado por nadie ${ }^{4}$.

Sería absurdo (y en cualquier caso intolerablemente inmodesto) intentar evaluar de manera completa la ambiciosa pretensión logicista de L. FERRAJOLI en un breve estudio como éste; o intentar — siguiendo el camino trazado por GÖDEL en la confutación de la teoría de RUSSELL- determinar si resulta exitosa o no.

El presente estudio tiene un objetivo mucho más modesto: presentar y examinar en detalle algunos aspectos fundamentales de la teoría lógico-jurídica de L. FERRAJOLI. Más en concreto, en la primera sección expondré algunas características principales de su lógica jurídica, haciendo hincapié, en particular, en los diferentes estatus que se le pueden adscribir. En la segunda sección presentaré sucintamente algunas observaciones críticas acerca de los postulados de consistencia y completitud, sobre los cuales la teoría de FERRAJOLI está fundada. En la tercera - y última- sección trataré brevemente de las normas implícitas (i. e. derivadas) y su estatus en los sistemas jurídicos organizados en diferentes niveles jerárquicos.

\section{TRES PERSPECTIVAS ACERCA DE LA LÓGICA JURÍDICA}

En la obra de FERRAJOLI, cabe identificar al menos tres tipos de perspectiva lógico-normativa: a) la lógica de la teoría axiomática del Derecho; b) la lógica del Derecho, y la lógica de los juristas (cuyo discurso, supuestamente, está compuesto por aserciones jurídicas, concebidas como proposiciones descriptivas que versan sobre el contenido del Derecho). FERRAJOLI se refiere expresamente a la primera y a la segunda perspectivas; en cambio, la tercera surge de una cierta interpretación de las fórmulas deónticas, que no es mencionada en Principia Iuris, pero que parece

1 Ibid., Teoria assiomatizzata del diritto. Parte generale, Milano, Giuffrè, 1970.

2 Ibid., Principia Iuris. Teoria del diritto e della democrazia, Bari-Roma, Laterza, 2007. Las referencias a estas obras aparecerán entre paréntesis en el texto, mientras que en las notas a pie de página serán precedidas por la abreviación «PI».

Vid. B. Russell, Introduction to Mathematical Philosopby, London, George Allen \& Unwin, 1919, cap. 18. Vid. también W. V. O. QuINE, «New Foundations for Mathematical Logic», en American Mathematical Quarterly, 1937.

4 Cfr. P. ODIFREDDI, Introduzione a B. Russell, Introduzione alla filosofia matematica, Milano, Longanesi, 2007, 7-8. 
claramente disponible si se considera la historia de la lógica deóntica y de la teoría jurídica ${ }^{5}$.

Examinemos en detalle estas tres perspectivas.

\subsection{La sintaxis de la teoría del Derecho}

El primer tipo de perspectiva lógica asumida por FERRAJOLI consiste en la elaboración de los instrumentos lógicos necesarios para construir su propia teoría jurídica. Su concepción es axiomática: parte de ciertas asunciones y ciertos postulados y luego deriva todas sus consecuencias lógicas (o, por lo menos, las consecuencias más relevantes). Cabe observar que FERRAJOLI considera la consistencia como el principal requisito para la «felicidad» de su teoría jurídica, siendo los otros requisitos ${ }^{6}:$ i) a exactitud semántica de los términos que la componen; ii) a transparencia acerca de las elecciones pragmáticas o explicativas que subyacen a la teoría, y iii) el carácter fructífero de la teoría, que admite el control de su misma consistencia y requiere expresamente ulteriores desarrollos teóricos bajo la forma de nuevos teoremas todavía no derivados. FERRAJOLI da por sentado que su teoría no es completa (ya que muchas nuevas derivaciones pueden ser realizadas en su ámbito ${ }^{7} \mathrm{y}$, por consiguiente, no caracteriza la completitud (sino más bien la «integrabilidad» 0 «completabilidad») como un criterio para el éxito de la misma teoría ${ }^{8}$.

La teoría de FERRAJOLI se funda en predicados primitivos y predicados definidos, que son elaborados mediante la combinación de los términos primitivos. Las conectivas lógicas usuales son empleadas de manera bastante tradicional ${ }^{9}$.

Sin embargo, es importante observar que la conectiva disyuntiva « $\backslash »$ es usada de manera ambigua en la teoría (más frecuentemente como una disyunción incluyente, pero a veces también como disyunción excluyente) ${ }^{10}$, según el significado de los términos categoremáticos (o sea, de los predicados).

Esto conlleva que todas las conectivas cuyas definiciones son, obviamente, correlacionadas entre sí, no deben ser entendidas como conectivas meramente sintácticas,

5 Vid. E. Bulygin, Normas, proposiciones normativas y enunciados jurídicos, en C. E. AlCHOURRón y É. Bulygin, Análisis lógico y Derecho, Madrid, Centro de Estudios Constitucionales, 1991.

${ }^{6}$ Ibid. «Principia iuris. Una discusión teórica», en Doxa, núm. 31, 2008 (desde ahora en adelante: «DT»), 396.

7 Cabe notar que la teoría ni siquiera puede ser completa, tratándose de un sistema que contiene una lógica predicativa de segundo orden. Lo que parece razonable requerir es la completud acerca de las consecuncias (consideradas) relevantes.

8 PI, III, 4-5; DT, 396.

9 Las conectivas son la siguientes:

«\»(«no»: negación).

$\ll \& »$ («y»: conjunción).

$\ll \vee »(\ll \mathrm{O} »$ : disyunción).

« («si... entonces»: implicación material).

«↔» («si y sólo si... entonces»: equivalencia material).

A decir verdad, FERRAJOLI usa «`» por la conjunción y «झ» por la equivalencia, pero aquí prefiero utilizar otra simbolización. Es preciso también señalar que FERRAJOLI emplea el símbolo ortogonal « $\perp »$ para referirse a la negación de un argumento (i. e. de un contenido proposicional). PI, I, 148 .

${ }_{10}$ Para un caso claro (y expresamente reconocido) de ambigüedad de la disyunción, vid., por ejemplo, 
sino, dicho de algún modo, como conectivas «mixtas» (a la vez, sintácticas y semánticas). Esto genera una importante dificultad acerca de la fundación axiomática de la teoría como meramente sintáctica ${ }^{11}$, y parece contradecir el requisito de la exactitud de los términos de la teoría que, como hemos visto, es uno de los criterios principales para evaluar el valor científico de la misma.

La teoría presenta cuatro niveles de axiomas y reglas de inferencia. Está basada, más en concreto, sobre la lógica proposicional, la lógica de predicados ${ }^{12}$ y la lógica modal ${ }^{13}$ (en particular, el sistema S5 complementado con la formula de BARCAN) ${ }^{14}$.

Finalmente, un axioma particular de carácter metateórico (llamado «PM»), introducido específicamente en la teoría axiomatizada, establece lo siguiente (III, 19-20):

$$
(\mathrm{y})(\mathrm{Py} \leftrightarrow \mathrm{M}(\exists \mathrm{x}) \text { Pyx })
$$

A saber: si y sólo si un predicado es monádico en relación con un cierto argumento $y$, entonces es posible que exista otro argumento $x$ acerca del cual $\mathrm{P}$ funciona como un predicado diádico. Por ejemplo, «si $y$ es una facultad, entonces es la facultad de un posible argumento, y viceversa» (III, 20).

\subsection{La lógica del Derecho}

Acerca de la lógica propia del Derecho, FERRAJOLI distingue, haciéndose eco de la conocida terminología de KELSEN, entre sistemas normativos estáticos y sistemas normativos dinámicos ${ }^{15}$. Los sistemas estáticos son aquellos en los que «una norma existe y es válida si puede ser deducida de otras normas del mismo sistema» (I, 114). Sistemas dinámicos son aquellos en los que la existencia de una norma depende del hecho de haber sido promulgada por una autoridad normativa competente (I, 115).

FERRAJOLI instituye una equivalencia entre el «estado meramente legislativo» y un sistema normativo totalmente dinámico, mientras que el estado constitucional contemporáneo es equiparado a un sistema normativo mixto, a la vez estático y dinámico (veremos más adelante la articulación de este concepto).

11 Vid., e. g., PI, I, 52: «La entera teoría [...] es un sistema, cuya validez depende únicamente de su consistencia interna, prescindiendo de sus directas y específicas referencias semánticas».

12 Es importante enfatizar el hecho de que las modalidades deónticas sean concebidas como predicados. Dichas modalidades son: obligatorio («Permitido $\phi y$ no permitido no- $\phi »)$ ); prohibido (permitido no- $\phi y$ no permitido $\phi »)$; facultativo («permitido $\phi y$ permitido no- $\phi »)$.

13 Según Ferrajoli (DT, 407, especialmente nota 11), la lógica modal ha hecho su teoría mucho más potente que su primera formulación de 1970, ya que ha hecho posible «dar cuenta del carácter sobre todo deóntico de los fenómenos jurídicos».

14 Por consiguiente, los axiomas de la lógica modal de FERRAJOLI son los siguientes («L» representa el operador alético «necesario» $\mathrm{y} \ll \mathrm{M} \gg$ el operador alético «posible»):

A8 Lp $\rightarrow$ p.

A9 $\mathrm{L}(\mathrm{p} \rightarrow \mathrm{q}) \rightarrow(\mathrm{Lp} \rightarrow \mathrm{Lq})$.

$\mathrm{A} 10 \mathrm{Mp} \rightarrow \mathrm{LMp}$.

A11 (x)LPx $\rightarrow$ L(x)Px.

Además, hay la regla de inferencia NEC (es decir: la «regla de necesitación»), que establece que «Si A es una tesis lógica, entonces $\mathrm{L}(\mathrm{A})$ es una tesis lógica».

15 Para una distinción clara de los dos criterios (junto a sus posibles combinaciones), vid. R. CARACCIOLO, El sistema jurídico. Problemas actuales, Madrid, Centro de Estudios Constitucionales, 1988. 
De todos modos, lo que es importante subrayar aquí es que, en los sistemas del primer tipo, los principios de no-contradicción y de completitud son leyes lógicas internas o incluso constitutivas del sistema (las que FERRAJOLI llama principia iuris et in iure), mientras que son externos en relación a los sistemas dinámicos (I, 172). En estos últimos, son principia iuris tantum, es decir «modelos normativos impuestos al Derecho vigente por la lógica y la teoría jurídica» (I, 117).

Por consiguiente, FERRAJOLI mantiene que las antinomias y las lagunas normativas son imposibles en los sistemas normativos estáticos, ya que «cuando una norma es (considerada) incompatible con otra, una de estas dos normas debe ser excluida como inválida, o mejor inexistente, bajo la amenaza de la inconsistencia del sistema» (I, 115).

De ahí que los postulados P1 y P2 —que respectivamente expresan (como veremos más adelante) el principio de no-contradicción y el principio de completitud- habrían de entenderse como descriptivos en relación con los sistemas estáticos, mientras habrían de considerarse como prescriptivos en relación con los sistemas dinámicos.

Otra dicotomía es introducida por FERRAJOLI en relación con la consistencia y la completitud del Derecho. En los sistemas jurídicos con un solo nivel normativo (típicos de los que FERRAJOLI llama «estados legislativos»), lagunas y antinomias no existen, ya que los conflictos y las lagunas aparentes pueden siempre ser solucionados según los criterios proporcionados por el mismo sistema. En un cierto sentido, el Derecho reconoce los principios lex posterior y lex specialis, y el razonamiento analógico, como reglas de inferencia lógicamente válidas. Manifiestamente, esto parece ser incompatible con las reglas de inferencia establecidas por la teoría de FERRAJOLI. Además, parece que estos criterios, más allá de no poder solucionar todos los posibles defectos sistémicos, no son conceptualmente necesarios (a diferencia de las reglas lógicas).

Sin embargo, podemos entender, de manera más caritativa, que FERRAJOLI mantiene que en los sistemas jurídicos de un solo nivel los intérpretes siempre pueden solucionar los defectos sistémicos internamente, mientras que en los sistemas articulados en diversos niveles (el caso paradigmático de los cuales serían los «estados constitucionales»), las lagunas y las antinomias no son solucionables mediante interpretación: necesitan la intervención ab extra de las autoridades legislativas y jurisdiccionales competentes (I, 13).

De todos modos, según FERRAJOLI, las lagunas y las antinomias «verdaderas» son posibles únicamente en los sistemas jurídicos articulados en más de un nivel, ya que dichos defectos son definidos, respectivamente, como la omitida producción de una norma requerida por el nivel normativo superior, y la producción de una norma en colisión con lo que dispone el nivel normativo superior (I, 685-686). Es preciso enfatizar que, según FERRAJOLI, ambos defectos sistémicos proceden de la violación de una norma acerca de la producción de normas jurídicas (que tienen como objeto el contenido de normas que deben, o no deben, ser producidas por el legislador).

\subsection{La lógica de los juristas}

Como observa GuASTINI ${ }^{16}$, Principia Iuris dice muy poco acerca del razonamiento de los juristas. Es más, lo que es aún más importante para la perspectiva escogida en

${ }_{16}$ R. GuAstinI, «Algunos aspectos de la metateoría de Principia Iuris», en Doxa, núm. 31 (2008): 254-255. 
este ensayo es que FERRAJOLI ni siquiera menciona la distinción fundamental entre normas y proposiciones normativas (es decir, proposiciones descriptivas que versan sobre la pertenencia de las normas a los sistemas jurídicos), siendo estas últimas supuestamente usadas por la «ciencia jurídica» para describir el Derecho vigente.

Una razón que explicaría esto puede ser que FERRAJOLI asume, como veremos con mayor detenimiento más adelante, que los sistemas jurídicos deben ser completos y consistentes. Bajo esta asunción, en efecto, el comportamiento lógico de normas y proposiciones normativas es realmente idéntico. Cuando se dan estas condiciones, usando proposiciones normativas es posible reflejar, por así decirlo, el comportamiento lógico de las normas. Esto, a su vez, tiene la consecuencia importante de hacer menos urgente distinguir entre permisión fuerte y permisión débil. Es decir, entre la proposición que afirma el hecho de que una cierta norma, que autoriza una cierta conducta, pertenece a un determinado sistema jurídico, y la proposición que describe el hecho de que una cierta norma, que prohíbe una cierta conducta, no pertenece a un determinado sistema jurídico.

Sin embargo, FERRAJOLI es bastante claro al afirmar que, como cuestión empírica, «la completitud y la consistencia [...] son virtualmente excluidas en el Estado constitucional de Derecho» (I, 443), y que «es inevitable» que «en un mismo orden jurídico positivo existan normas contradictorias» (I, 117). Esto significa que las normas y las proposiciones normativas no pueden ser lógicamente equiparadas, y que diferentes tipos de permisión deben ser admitidos por la teoría. Si esto es correcto, entonces es posible leer las fórmulas de la teoría axiomática de FERRAJOLI de dos maneras distintas: o bien como fórmulas que expresan normas (o, mejor dicho, ideales regulativos) o bien como fórmulas que expresan proposiciones acerca de normas. La segunda lectura es lo que llamo «lógica de los juristas».

Sin embargo, interpretadas como proposiciones normativas, muchas fórmulas — como por ejemplo «(x) ((ヨy') PERy’x $\rightarrow \neg(\exists y ”)$ PRHy”x)» que asevera que un argumento no puede ser simultáneamente permitido y prohibido- afirmaría por lo general, según los criterios ofrecidos por FERRAJOLI, una proposición falsa en relación con los estados constitucionales, y una proposición verdadera en relación con los estados legislativos. Más en general, podemos decir que los valores de verdad de dichos enunciados pueden ser determinados sólo como una cuestión empírica (y por ende no conceptual), ya que las proposiciones normativas son, por definición, relativas a un cierto sistema normativo.

\section{UN ANÁLISIS DE LOS POSTULADOS DE COMPLETITUD Y DE CONSISTENCIA}

Toda la sofisticadísima teoría axiomática concebida por FERRAJOLI está basada en dos axiomas, que resultan algo dudosos.

El postulado P2 de la lógica jurídica de FERRAJOLI afirma que «cualquier conducta supone alguna modalidad deóntica de la que constituye el argumento» ${ }^{17}$. En fórmulas:

$$
\text { P2. }(\mathrm{x})(\mathrm{CONx} \rightarrow(\exists \mathrm{y}) \mathrm{MODyx})
$$

17 PI, I, 67; III, 22. 
Como es fácil ver, P2 dispone que el Derecho sea necesariamente completo, ya que es imposible que pueda haber una conducta sin que haya una correspondiente modalidad que la califique:

$$
[\mathrm{T} 2 \alpha](\mathrm{x}) \neg(\mathrm{CONx} \& \neg((\exists \mathrm{y}) \mathrm{MODyx}))
$$

Entendido como un ideal regulativo, dicho enunciado dispone que las autoridades normativas deban producir sistemas normativos completos. En cambio, si se entiende como una proposición normativa, afirma que una cierta regla, la cual califica jurídicamente todas las conductas que no son calificadas por las demás normas jurídicas, pertenece al sistema jurídico que se pretende describir ${ }^{18}$.

El primer postulado, a su vez, es el siguiente:

$$
\text { P1. (x) }(\neg \text { PERx } \rightarrow \text { PER } \perp x)
$$

El postulado P1 no es fácil de interpretar, a pesar de las apariencias.

Según una primera interpretación, no es otra cosa que el clásico principio «Obligatorio implica permitido», como muestra la siguiente formula (obtenida mediante la mutua sustitución de $\langle\mathrm{x} \gg \mathrm{y}\langle\perp \mathrm{x}\rangle)$ :

$$
\mathrm{T} 1.1(\mathrm{x})(\neg \mathrm{PER} \perp \mathrm{x} \rightarrow \mathrm{PERx})
$$

No diversamente de P2, la interpretación de dicho principio cambia según su interpretación en términos de normas o de proposiciones normativas.

En los sistemas estáticos, por definición, cuando « $\neg \mathrm{PER} \perp \mathrm{x}$ » es válido, «PERx» debe también ser válido. En cambio, en los sistemas dinámicos, no es el caso que mandar $x$ implique permitir $x$ : una autoridad normativa debe siempre promulgar una norma permisiva, si quiere autorizar $x$. Se puede dudar que dicho principio rija en los estados constitucionales, que son considerados por FERRAJOLI como eminentemente dinámicos. Como sostiene GUASTINI ${ }^{19}$, puede ser más bien interpretado como una «recomendación», destinada al legislador, de promulgar todas las normas lógicamente implicadas por las normas de nivel constitucional ${ }^{20}$.

En cambio, en relación con las proposiciones normativas, tenemos que distinguir entre permisión fuerte y débil. Una vez introducida dicha distinción, cabe observar que T1.1 quizá sea válido respecto de la permisión fuerte, pero claramente no lo es en relación con la permisión débil. Del hecho de que algo haya sido mandado, efectivamente, no se sigue que lo mismo no haya sido prohibido (sin presuponer anteriormente, mediante una petición de principio, la consistencia del sistema). A este respecto, cabe observar que, según el mismo sistema lógico de FERRAJOLI, una situación de permisión débil garantiza de por sí la consistencia del sistema. Esto se debe a la circunstancia relevante de que «el mero hecho de que haya una proposición que no pertenece al sistema garantiza ya su coherencia, ya que de un sistema incoherente puede derivarse

18 Se observa que en los «estados legislativos», que pueden ser caracterizados como estados de Derecho, dicha regla es normalmente dúplice: la norma que impone o autoriza el razonamiento analógico por el Derecho civil, y la llamada «norma general exclusiva» por el Derecho penal.

19 R. GuASTINI, «Algunos aspectos de la metateoría de Principia Iuris», cit., 258.

20 Elaboraré este argumento con más detenimiento en la sección final. 
cualquier proposición» ${ }^{21}$. Esto significa, a su vez, que si FERRAJOLI sostiene que los sistemas constitucionales son necesariamente inconsistentes (como se desprende de las aserciones de FERRAJOLI en I, 117), entonces debe aceptar el teorema, derivable de su propia teoría, según el cual dichos sistemas son necesariamente completos, porque todo se sigue de un sistema inconsistente. Sin embargo, si esto es correcto, entonces P1 es necesariamente verdadero en relación con los sistemas constitucionales y, por consiguiente, no puede funcionar como un ideal regulativo.

Como es fácil ver, otro teorema se sigue de P1:

\section{$\mathrm{T} 1.2(\mathrm{x})(\mathrm{PERx} \vee \mathrm{PER} \perp \mathrm{x})$}

E. Bulygin y J. J. Moreso han sostenido, con razón, que P1 no es otra cosa que un teorema de P2, como T1.2 permite demostrar ${ }^{22}$. De ahí que los primeros dos postulados no serían independientes y los fundamentos de la teoría deberían ser revisados de consecuencia.

Según una cierta interpretación, T1.2 parece prima facie ser compatible con la presencia de lagunas en el Derecho, ya que afirma que $x$ está permitido, o no- $x$ está permitido, o ambos están permitidos. En otras palabras, niega que ambos estén prohibidos (lo que afirma el teorema T1.3). El problema con T1.2 es que es nuevamente ambiguo ya que no sabemos si expresa una norma o una proposición normativa.

Si expresa una norma, BulYGIN y MORESO tienen razón en enfatizar que P1 necesariamente conlleva la completitud de los sistemas jurídicos. Según T1.2, en efecto, si no- $x$ no está permitido, se sigue (por silogismo disyuntivo) que $x$ está permitido; si $x$ no está permitido, se sigue (de nuevo por silogismo disyuntivo) que no- $x$ está permitido; si ambos están permitidos, se sigue manifiestamente que el sistema dispone la facultad de realizar $x$. El sistema es completo ya que contiene, alternativamente, la obligación, la prohibición, o la facultad de hacer $x$ (como afirma el teorema T1.47) ${ }^{23}$.

En cambio si T1.2 se lee como una proposición normativa, se sigue que la proposición que versa sobre la prohibición de no- $x$ en un cierto sistema no implica la verdad de la proposición que versa sobre la permisión fuerte o débil de $x$, y la proposición que asevera el hecho de que $x$ está prohibido no implica nada en relación con la permisión fuerte o débil de no- $x$, de forma que la aserción de FERRAJOLI según la cual «"permitido" es de por sí interpretable como "permiso débil" ${ }^{24}$ suena bastante oscura. Según esta última lectura, en efecto, parecería que la permisión débil implicara la permisión fuerte, lo que se puede asumir si y sólo si hay una norma de clausura a este efecto (es decir, si el sistema es completo: lo que debería ser probado y no asumido como premisa del razonamiento, si se quiere evitar caer en una petición de principio).

Otro teorema de P1 afirma lo siguiente:

$$
\mathrm{T} 1.3(\mathrm{x}) \neg(\neg \mathrm{PERx} \& \neg \mathrm{PER} \perp \mathrm{x})
$$

21 C. E. Alchourrón y E. Bulygin, «Fundamentos pragmáticos para una lógica de normas», en C. E. Alchourrón y E. Bulygin, Análisis lógico y Derecho, cit., 165.

22 Cfr. E. Bulygin, «Algunas reflexiones sobre lagunas y antinomias en Principia Iuris», y J. J. MoRESO, «Ferrajoli o el constitucionalismo optimista», ambos en Doxa, núm. 31, 2008. Vid. también la respuesta de FERRAJOLI (a mi parecer, no muy convincente) en DT: 408-410.

23 PI, III: 46.

24 DT: 408, nota 11. 
Esto significa que un cierto argumento no puede ser, a la vez, obligatorio y prohibido. Esto es, estrictamente hablando, el principio de no-contrariedad. Pero se puede demostrar que dicho principio se deriva fácilmente del principio de no-contradicción: $\ll(\mathrm{x}) \neg(\neg \mathrm{PERx} \& \mathrm{PERx}) »$.

Sin embargo, aquí de nuevo nos enfrentamos con una ambigüedad: interpretado como una norma, dicho principio significa que el legislador no debe promulgar normas que sean incompatibles con otras normas (superiores). Entendido como una proposición acerca de normas, describe las condiciones bajo las cuales el sistema es consistente, sin excluir la posibilidad de sistemas incoherentes ${ }^{25}$.

Acerca de la posibilidad de conseguir simultáneamente la consistencia y la completitud (i. e. de satisfacer P1 y P2) de un sistema con distintos niveles jerárquicos es preciso realizar una observación no secundaria.

Como ya hemos visto, P2 puede ser leída como la recomendación a la asamblea constituyente (o al legislador) de promulgar una regla de clausura, que hace que el sistema jurídico correspondiente sea completo para todo caso posible.

En la literatura acerca de los sistemas normativos ${ }^{26}$, se opina que una regla de clausura exitosa deba poseer las dos siguientes características: a) debe clausurar el sistema, en el sentido de que debe ofrecer una respuesta jurídica a toda posible conducta en relación con toda posible clase de «hechos operativos»; b) debe preservar la consistencia del sistema: si una regla de clausura no es capaz de preservar la consistencia, clausura efectivamente el sistema pero al precio de hacerlo inservible para fines prácticos.

En cuanto a la posible formulación de posibles reglas de clausura generales, hay al menos tres opciones: i) «Todo lo que no está expresamente calificado está permitido»; ii) «Todo lo que no está expresamente calificado está prohibido»; iii) «Todo lo que no está expresamente calificado es obligatorio». Las opciones ii) y iii) deben ser descartadas por razones lógicas: ambas producen antinomias y, por consiguiente, hacen inconsistente un sistema que era originariamente consistente.

De ahí que la única regla de cierre — entre las que hemos mencionado- que puede ser usada, de manera exitosa, es una norma general permisiva.

Sin embargo, aunque las reglas de clausura permisivas son totalmente seguras en relación con sistemas normativos categóricos (es decir, sistemas compuestos únicamente por normas categóricas), producen algunos problemas si se aplican a sistemas normativos hipotéticos. Y el Derecho es usualmente (si no siempre) compuesto por (al menos un cierto número) de normas hipotéticas: es un sistema normativo hipotético.

Ahora bien, ALCHOURRÓN y BULYGin han demostrado que no hay reglas de clausura generales de un sistema normativo hipotético que no creen contradicciones que no estaban presentes en dicho sistema normativo antes de la promulgación de dicha regla ${ }^{27}$.

25 C. E. Alchourrón y E. Bulygin, «Fundamentos pragmáticos para una lógica de normas», cit., 162.

26 El locus classicus es C. E. AlchOURRÓn y E. BulYgIn, Introducción a la metodología de las ciencias jurídicas y sociales, Buenos Aires, Astrea, 1975, 189-196.

27 Ibid. 
Esto se debe a dos circunstancias notables ${ }^{28}$ : a) las reglas de clausura se aplican, inter alia, a clases de casos que son menos específicos que los casos relevantes; $b$ ) aplicando la regla lógica del refuerzo del antecedente — que es aceptada por FERRAJOLI mediante la ley lógica L4.31 «(p $\rightarrow$ q) \& p) $\rightarrow$ q»- a dichas clases de casos menos específicos obtenemos, en muchas ocasiones, soluciones que son incompatibles con las que están correlacionadas con clases de casos menos específicos.

Por consiguiente, las reglas de clausura, como las que hemos analizado hasta ahora, cierran el sistema al precio de hacerlo indeterminado: introducen antinomias en un sistema originariamente consistente.

Pues bien, a los efectos de formular exitosamente reglas generales de clausura, las autoridades normativas deben restringir su aplicación a la clase de casos que han identificado como relevantes.

Esto, a su vez, plantea algunas cuestiones importantes acerca la interacción de los dos niveles de legalidad (el constitucional y el legislativo) sobre la cual FERRAJOLI construye su teoría, ya que la clausura del sistema puede variar en relación con el nivel de relevancia escogido. Y esto es así porque una clausura general al nivel de relevancia R1 (por ejemplo, el nivel constitucional) puede producir bastantes antinomias respecto al nivel de relevancia R2 (e. g., el nivel legislativo). En efecto, muchas conductas que serían calificadas como permitidas por la regla de clausura perteneciente al nivel R1 podrían ser tranquilamente calificadas como prohibidas por el nivel R2 (y viceversa).

\section{NORMAS DERIVADAS Y AUTORIDADES NORMATIVAS}

En esta última sección, quiero solamente formular una observación muy breve acerca de las normas implícitas en la teoría de FERRAJOLI.

Como hemos visto, una de las tesis principales de Principia Iuris es que, en los sistemas constitucionales, el legislador no debe promulgar normas incompatibles con la constitución. Hemos visto también que un sistema jurídico debe ser completo, de acuerdo con el segundo postulado de FERRAJOLI. Hemos también considerado algunas razones para sostener que esto sólo se puede hacer mediante una regla de clausura permisiva concerniente el solo nivel constitucional (de otra manera el sistema colapsaría bajo el peso de nuevas contradicciones). Sin embargo, esto significa que no hay ningún espacio vacío de Derecho constitucional: todo está establecido por la constitución.

Si esto es correcto, el legislador no tiene ningún espacio libre para legislar ${ }^{29}$. Se debe limitar a: i) primero, derivar todas las normas implicadas por las normas consti-

28 Las circunstancias mencionadas en el texto se dan si y sólo si uno se compromete con la monotonía: lo que se ha convertido en algo bastante controvertido en la lógica y en la teoría jurídica. Acerca de este punto, vid. C. E. Alchourrón, Fundamentos para una teoría general de los deberes, Madrid, Marcial Pons, 2010, especialmente 102 ss. De todos modos, como se ha señalado en el texto, FERRAJOLI está comprometido con la monotonía a través de L4.31.

${ }_{29}$ Esto implica que, contrariamente a lo que se sostiene usualmente en la teoría jurídica, FERRAJOLI mantiene un modelo conjuntivo de la validez, que requiere a la vez legalidad y deducibilidad para que una norma sea válida: es decir, el modelo M3 de la conocida clasificación ofrecida por R. CARACCIOLO, El sistema jurídico, cit., 60 . 
tucionales; ii) segundo, cuando una derivación formal directa es (considerada) imposible, determinar los medios mediante los cuales alcanzar el fin establecido por las normas constitucionales ${ }^{30}$. En ambos casos, el sistema parece ser eminentemente estático y su faceta dinámica consistiría únicamente en el mecanismo institucional que debería permitir desarrollar (las consecuencias lógicas de) dicho sistema. Malvadamente, uno tiene la tentación de preguntar: ¿Por qué no dejar esta tarea a lógicos bien entrenados, en lugar de dejarla a legisladores que a menudo ignoran hasta los instrumentos más básicos de la lógica deductiva?

${ }^{30}$ Como he tratado de argüir en G. B. RatTI, «A Note on the Logical Structure of Legal Principles», in Analisi e diritto, 2009, las directrices pueden ser reconstruidas como normas que disponen obligaciones alternativas cerradas. Si esto es así, el legislador debería limitarse a elegir un cierto medio para alcanzar el fin identificado por una cierta directriz en el ámbito de un conjunto de medios previamente identificables. 